\title{
SRB MEASURES FOR AXIOM A ENDOMORPHISMS
}

\author{
Mariusz Urbanski And Christian Wolf
}

\begin{abstract}
Let $\Lambda$ be a basic set of an Axiom A endomorphism $f$ on a $n$-dimensional compact Riemannian manifold. In this paper, we provide equivalent conditions for the existence of an SRB measure on $\Lambda$. In particular, we show that under the assumption that the closure of the postcritical set of $f$ is disjoint from $\Lambda$, the existence of an SRB measure is equivalent to the condition that the stable set of $\Lambda$ has packing dimension $n$. Our approach uses the theory of thermodynamic formalism. In particular, we consider the topological pressure on the inverse limit space.
\end{abstract}

\section{Introduction}

In the theory of dynamical systems much research has been focused on the statistical properties of a given system with respect to an invariant probability measure. However, for many systems the set of invariant measures is rather large. This raises the question, which invariant measure is the natural choice to study. From an applications point of view, the only measures which can actually be "observed" have the property that the set of points, whose orbit distribution coincides with the measure, has positive Lebesgue measure. Measures with this property are called physical measures (see section 2.3 for the precise definition). Another important class of measures are the so-called SRB-measures (standing for Sinai, Ruelle, Bowen). These measures are characterized by the property that the corresponding conditional measures on the unstable manifolds are absolutely continuous with respect to the Lebesgue measure. It turns out that for many classes of systems the notion of a physical measure coincides with that of an SRBmeasure. We refer to [11] for more details about SRB and physical measures. The existence of SRB-measures is fully understood only in the case of hyperbolic sets of $C^{2}$-diffeomorphisms on compact Riemannian manifolds. For this class of systems, it follows from the work of R. Bowen and D. Ruelle that the existence of an SRB-measure is equivalent to $\Lambda$ being an attractor (see for instance [11] and the references therein). Moreover, SRB measures are physical measures and vice versa. An a priori weaker but still equivalent condition in terms of Hausdorff dimension of stable sets was recently derived by R. Shafikov and C. Wolf, see $[9]$.

Received March 18, 2004.

2000 Mathematics Subject Classification. Primary: 37C45, 37DXX.

Key words and phrases. SRB-measure, physical measure, Axiom A endomorphism. 
In the case of Axiom A endomorphisms the theory of SRB-measures is to date less complete as in the diffeomorphism case. M. Qian and S. Zhu introduced in [7] the notion of an SRB-measure ${ }^{1}$ in the case of non-uniformly hyperbolic $C^{2}$ endomorphisms on compact Riemannian manifolds by using the inverse limit space construction. Applying their results to the Axiom A case (also using results from [6]) they proved that if $\Lambda$ is an attractor, then there exists a unique SRB-measure $\mu$ supported on $\Lambda$. Furthermore, $\mu$ is a physical measure, and Pesin's entropy formula holds. We refer to [7] for more details.

The main purpose of this paper is to continue the study of SRB-measures for Axiom A endomorphisms. In particular, we provide a classification for the existence of an SRB-measure in terms of the packing dimension $\mathrm{PD}\left(\mathrm{W}^{\mathrm{s}}(\Lambda)\right)$ of the stable set of $\Lambda$. Our main result is the following (see Theorems 8 and 10 in the text).

Theorem 1. Let $f$ be a $C^{2}$ Axiom A endomorphism on an n-dimensional compact Riemannian manifold $M$, and let $\Lambda$ be a basic set of $f$ such that $\overline{\mathrm{PC}(f)} \cap \Lambda=$ $\varnothing$. Then the following are equivalent.

(i) $\mathrm{PD}\left(\mathrm{W}^{\mathrm{s}}(\Lambda)\right)=\mathrm{n}$;

(ii) $\Lambda$ is an attractor;

(iii) $f$ admits an $S R B$ measure on $\Lambda$;

(iv) $f$ admits a physical measure on $\Lambda$;

(v) $\Lambda$ admits an invariant measure satisfying Pesin's entropy formula.

(vi) $W^{s}(\Lambda)$ has positive Lebesgue measure.

Here $\mathrm{PC}(f)$ denotes the postcritical set of $f$ (see section 3 for the definition). Note that $(i) \Leftrightarrow(i i)$ has not been known before even for Axiom A diffeomorphisms. Since there exist $C^{1}$ horseshoes with positive Lebesgue measure (see [1]), the hypotheses that $f$ is a $C^{2}$ map is crucial in Theorem 1. It turns out that the conditions $(i i),(i i i),(i v)$ and $(v)$ in Theorem 1 are still equivalent under the weaker assumption that $\Lambda$ does not contain any critical point of $f$ (see Theorem 8). One consequence of Theorem 1 is that any basic set of an Axiom A endomorphism, which is not an attractor, must have packing dimension strictly smaller than $n$. An even stronger result is given in Corollary 10 where we derive an explicit upper bound for the packing dimension of $W^{s}(\Lambda)$. This bound can be shown to be strictly smaller than $n$.

It is known that a hyperbolic set of a $C^{2}$ diffeomorphism has either non-empty interior or its upper box dimension is strictly smaller than $n$ (see [9]). It would be interesting to know whether an analogous dichotomy result holds in the case of Axiom A endomorphisms. The difficulty to prove such a result comes from the fact that the map is not necessarily invertible. In particular, the methods used in [9] are not applicable.

This paper is organized as follows. In Section 2 we present the required ergodic-theoretical results for Axiom A endomorphisms parallel to the theory

\footnotetext{
${ }^{1}$ More precisely, Qian and Zhu defined measures having the SRB property. In light of our Theorem 1, we will call these measures simply SRB measures.
} 
for diffeomorphisms developed by R. Bowen and D. Ruelle. Finally, in Section 3 we discuss various equivalent conditions to the existence of an SRB-measure.

\section{Ergodic theory for Axiom A endomorphisms}

In this section, we present some ergodic-theoretical results for hyperbolic endomorphisms. In particular, we study the thermodynamic formalism parallel to the corresponding theory of hyperbolic diffeomorphisms, which was developed by R. Bowen and D. Ruelle. The crucial difficulty comes from the fact that we have to consider the inverse limit space rather than just the hyperbolic set itself.

As a standing assumption we assume in this section that $f: M \rightarrow M$ is a $C^{2}$ endomorphism on an $n$-dimensional compact Riemannian manifold $M$, and $\Lambda \subset M$ is a completely $f$-invariant compact set, i.e., $f(\Lambda)=\Lambda=f^{-1}(\Lambda)$. Moreover, we assume that the set of critical points of $f$, denoted by $\mathcal{C}_{f}$, is disjoint from $\lambda$.

2.1. The inverse limit space. We define the inverse limit space of $f$ by

$$
M^{f}=\left\{\hat{x}=\left(x_{0}, x_{-1}, \cdots\right) \in M^{\mathbb{Z}^{-}}: f\left(x_{-i-1}\right)=x_{-i}, \text { for all } i \geq 0\right\} .
$$

Moreover, set $\Lambda^{f}=\left\{\hat{x}=\left(x_{0}, x_{-1}, \cdots\right) \in M^{f}: x_{0} \in \Lambda\right\}$. Given $K>1$, we define a metric

$$
d_{K}(\hat{x}, \hat{y})=\sum_{i \geq 0} \frac{d\left(x_{-i}, y_{-i}\right)}{K^{i}},
$$

on $M^{f}$, where $d$ denotes the Riemannian metric on $M$. The metric $d_{K}$ makes $M^{f}$ (respectively $\Lambda^{f}$ ) a compact metric space. It is easy to see that the topology on $M^{f}$ (respectively $\Lambda^{f}$ ) induced by the metric $d_{K}$ does not depend on the constant $K$. We denote by $\pi: M^{f} \rightarrow M$ the canonical projection defined by $\pi(\hat{x})=x_{0}$ and by $\hat{f}: M^{f} \rightarrow M^{f}, \hat{x} \mapsto\left(f\left(x_{0}\right), x_{0}, x_{-1}, \cdots\right)$ the left shift map. It is immediate that $\hat{f}$ is a homeomorphism. The tangent bundle over $\Lambda^{f}$ is defined by its fibers:

$$
T_{\Lambda^{f}}\left(\left(x_{n}\right)_{n}\right)=\left\{\left(\left(x_{n}\right)_{n}, v\right) \text { : with } v \in T_{x_{0}} M\right\} .
$$

2.2. Hyperbolicity. We say that $\Lambda$ is a hyperbolic set of $f$ if there exists a continuous splitting of the tangent bundle, $T_{\Lambda^{f}}(\hat{x})=E_{x_{0}}^{s} \oplus E_{\hat{x}}^{u}$ and constants $c>0, \lambda>1$, such that for all $x \in \Lambda$ and all $\hat{x}=\left(x_{0}, x_{-1}, \cdots\right) \in \Lambda^{f}$ :

(i) $d_{x} f\left(E_{x}^{s}\right) \subset E_{f(x)}^{s}$ and $d_{x_{0}} f\left(E_{\hat{x}}^{u}\right) \subset E_{\hat{f}(\hat{x})}^{u}$;

(ii) $\left\|d_{x} f^{n} v\right\| \leq c \lambda^{-n}\|v\|$ whenever $v \in E_{x}^{s}$ and $n \geq 0$;

(iii) $\left\|d_{x_{0}} f^{n} w\right\| \geq c^{-1} \lambda^{n}\|w\|$ whenever $w \in E_{\hat{x}}^{u}$ and $n \geq 0$.

We say that $f$ is an Axiom $A$ endomorphism if its non-wandering set $\Omega(f)$ is a hyperbolic set, and the set of periodic points of $f$, denoted by $\operatorname{Per}(f)$, is dense in $\Omega(f)$. By the spectral decomposition theorem (see for example [4]), $\Omega(f)$ is a finite union of basic sets. Let $\Lambda$ be a basic set of $f$, that is,

(i) $f \mid \Lambda$ is topologically transitive; 
(ii) $\Lambda$ is locally maximal, i.e., there exists a neighborhood $U$ of $\Lambda$ such that $\bigcap_{n=-\infty}^{\infty} f^{n}(U)=\Lambda$.

As in the case of hyperbolic diffeomorphisms, for all $x \in \Lambda, \hat{x} \in \Lambda^{f}$ and $\varepsilon>0$ small there exist stable and unstable manifolds $W_{\varepsilon}^{s}(x)$ and $W_{\varepsilon}^{u}(\hat{x})$ defined by

$$
W_{\varepsilon}^{s}(x)=\left\{y \in M: d\left(f^{n}(x), f^{n}(y)\right) \leq \varepsilon, \text { for all } n \geq 0\right\},
$$

$W_{\varepsilon}^{u}(\hat{x})=\left\{y \in M: y\right.$ has a prehistory $\hat{y}: d\left(x_{-n}, y_{-n}\right) \leq \varepsilon$, for all $\left.n \geq 0\right\}$.

Note that here $\varepsilon$ doesn't depend on $x \in \Lambda$. We say that $\Lambda$ is an attractor of $f$ if there are arbitrarily small neighborhoods $U$ of $\Lambda$ such that $f(U) \subset U$. This includes the case of Anosov endomorphisms (see [5]), for which the entire manifold $M$ is a hyperbolic set. We will need the following sufficient condition, parallel to Lemma 4.9 of [2], for a basic set to be an attractor.

Lemma 2. If $W_{\varepsilon}^{u}(\hat{x}) \subset \Lambda$ for some $\hat{x} \in \Lambda^{f}$ and for some $\varepsilon>0$, then $\Lambda$ is an attractor.

Proof. Let $\hat{x} \in \Lambda^{f}$ with $W_{\varepsilon}^{u}(\hat{x}) \subset \Lambda$. Then it follows that

$$
U_{\hat{x}}=\bigcup_{y \in W_{\varepsilon}^{u}(\hat{x})} W_{\varepsilon}^{s}(y)
$$

contains the point $x_{0}=\pi(\hat{x})$ in its interior. Indeed, by using the stable manifold theorem, one can construct a homeomorphism $h: U_{\hat{x}} \rightarrow B$, where $B$ is an open ball in $\mathbb{R}^{n}$ centered at the origin and $h\left(x_{0}\right)=0$.

Since periodic points of $f$ are dense in $\Lambda$, there exists a periodic point $p$ of $f$ in the set $\operatorname{Int}\left(U_{\hat{x}}\right)$. Denote $m$ to be the period of $p$. Let $\hat{p} \in \Lambda^{f}$ be the point formed by the infinite concatenation of the (finite) orbit of $p$. Since $p \in \operatorname{Int}\left(U_{\hat{x}}\right)$, there exists $\beta>0$ such that $W_{\beta}^{u}(\hat{p}) \subset \operatorname{Int}\left(U_{\hat{x}}\right)$. Take now an arbitrary point $z \in W_{\beta}^{u}(\hat{p})$. Then $z \in W_{\varepsilon}^{s}(y)$ for some $y \in W_{\varepsilon}^{u}(\hat{x}) \subset \Lambda$. In particular,

$$
y \in \Lambda \text { and } d\left(f^{n}(z), f^{n}(y)\right) \leq \varepsilon \text { for all } n \geq 0 .
$$

Since $z \in W_{\varepsilon}^{u}(\hat{p})$, there exists $\hat{z} \in \pi^{-1}(z)$ such that

$$
d\left(z_{-n}, p_{-n}\right) \leq \varepsilon \text { for all } n \geq 0 .
$$

Together with (5), and since $\Lambda$ is locally maximal, it follows that $z \in \Lambda$. Thus, we have proved that

$$
W_{\beta}^{u}(\hat{p}) \subset \Lambda
$$

We have

$$
\begin{aligned}
W_{2 \varepsilon}^{u}(\hat{p}) \subset W^{u}(\hat{p}) & =\left\{\xi \in M: \lim _{n \rightarrow \infty} d\left(\xi_{-n}, p_{-n}\right)=0 \text { for some } \hat{\xi} \in \pi^{-1}(\xi)\right\} \\
& =\bigcup_{j=0}^{\infty} f^{m j}\left(W_{\beta}^{u}(\hat{p})\right) .
\end{aligned}
$$

Given $\eta \in(0,2 \varepsilon)$, let

$$
\hat{W}_{\eta}^{u}(\hat{p})=\left\{\hat{\xi} \in \Lambda^{f}: d\left(\xi_{-n}, p_{-n}\right)<\eta \text { for all } n \geq 0\right\} .
$$


Then (see the proof of Theorem 3.4 in [4])

$$
\hat{W}^{u}(\hat{p})=\bigcup_{j=0}^{\infty} \hat{f}^{m j}\left(\hat{W}_{\eta}^{u}(\hat{p})\right)=\left\{\hat{\xi} \in \Lambda^{f}: \lim _{n \rightarrow \infty} d\left(\xi_{-n}, p_{-n}\right)=0\right\}
$$

It was established in the proof of Theorem 3.4 in [4] (spectral decomposition) that for some integer $l \geq 0$,

$$
\Lambda^{f}=\bigcup_{i=0}^{l} \hat{f}^{i}\left(\overline{\hat{W}^{u}(\hat{p})}\right)
$$

Put

$$
Y=\bigcup_{i=0}^{l} \hat{f}^{i}\left(\hat{W}^{u}(\hat{p})\right) \subset \Lambda^{f}
$$

We shall now show that

$$
\text { if } \hat{\xi}=\left(\xi_{0}, \xi_{-1}, \cdots\right) \in Y \text {, then } W_{\varepsilon}^{u}(\hat{\xi}) \subset \Lambda \text {. }
$$

Indeed, consider $y \in W_{\varepsilon}^{u}(\hat{\xi})$. Thus, there exists $\hat{y} \in \pi^{-1}(y)$ such that $d\left(\xi_{-n}, y_{-n}\right)$ $<\varepsilon$ for all $n \geq 0$. On the other hand, since $\hat{\xi} \in Y$, there exists $0 \leq k \leq l$ such that $\hat{\xi} \in \hat{f}^{k}\left(\hat{W}^{u}(\hat{p})\right)$. Therefore, (8) implies that $d\left(\xi_{-(k+n)}, p_{-n}\right) \rightarrow 0$ for $n \rightarrow \infty$. Hence $\hat{y} \in \hat{W}^{u}\left(\hat{f}^{k}(\hat{p})\right)$. Applying again (8), we obtain that $\left(y_{-j}, y_{-j-1}, \cdots\right) \in$ $\hat{W}_{\eta}^{u}(\hat{p})$ for some $j \geq 0$. Using (6) and (7) yields $y_{-j} \in \Lambda$. Therefore (10) follows from the fact that $\Lambda$ is a completely $f$-invariant set.

In view of (10), for every $\hat{\xi} \in Y$, the set $U_{\hat{\xi}}$ (see (4)) is well defined. We remark that by construction the set $Y$ results from the point $\hat{x}$ appearing in the assumptions of our lemma. Since, by (10), $U_{\hat{\xi}} \subset W_{\varepsilon}^{s}(\Lambda)$, we obtain that

$$
\bigcup_{\hat{\xi} \in Y} U_{\hat{\xi}} \subset W_{\varepsilon}^{s}(\Lambda)
$$

Since the function $\hat{z} \mapsto W_{\varepsilon}^{u}(\hat{z})$ and $x \mapsto W_{\varepsilon}^{s}(x), \hat{z} \in \Lambda^{f}, x \in \Lambda$, are continuous, it follows that the stable and unstable manifolds are uniformly transverse. More precisely, there exists $\alpha>0$ such that for all $\hat{\xi}=\left(\xi_{0}, \xi_{-1}, \ldots\right) \in \Lambda^{f}$ we have $\angle\left(W_{\varepsilon}^{s}\left(\xi_{0}\right), W_{\varepsilon}^{u}(\hat{\xi})\right)>\alpha$. We conclude that there exists $\delta>0$ such that $B\left(\xi_{0}, \delta\right) \subset U_{\hat{\xi}}$ for all $\hat{\xi} \in Y$. Since both sets $\Lambda$ and $\Lambda^{f}$ are compact and the projection $\pi: \Lambda^{f} \rightarrow \Lambda$ is a continuous surjection, it follows from (9) that $\pi(Y)$ is a dense subset of $\Lambda$. Therefore,

$$
B(\Lambda, \delta)=\bigcup_{\xi \in \pi(Y)} B(\xi, \delta) \subset \bigcup_{\hat{\xi} \in Y} U_{\hat{\xi}}
$$

Finally, by using (11), we conclude that $\Lambda$ is an attractor.

As a fairly straightforward consequence of this lemma, we get the following.

Lemma 3. Suppose $\Lambda$ is not an attractor. Then for every $\varepsilon>0$ there is $\gamma>0$ such that for every $\hat{x} \in \Lambda^{f}$ there exists $y \in W_{\varepsilon}^{u}(\hat{x}) \backslash B(\Lambda, \gamma)$. 
Proof. For every $\gamma>0$ let

$$
V_{\gamma}=\left\{\hat{x} \in \Lambda^{f}: W_{\varepsilon}^{u}(\hat{x}) \backslash B(\Lambda, \gamma) \neq \varnothing\right\} .
$$

The set $V_{\gamma}$ is open since the map $\hat{x} \mapsto W_{\varepsilon}^{u}(\hat{x})$ is continuous. It follows from Lemma 2 that $\bigcup_{\gamma>0} V_{\gamma}=\Lambda^{f}$. Since $\Lambda^{f}$ is compact and since the sets $V_{\gamma}$ are increasing as $\gamma \searrow 0$, we may conclude that $V_{\gamma}=\Lambda^{f}$ for all $\gamma>0$ small enough. This proves the lemma.

2.3. SRB-property, physical measures and Pesin's entropy formula. Let $\mathcal{M}$ denote the space of $f$-invariant probability measures on $\Lambda$, and $\mathcal{M}_{E}$ the subset of ergodic measures. Similarly, we denote by $\mathcal{M}^{f}$ the space of $\hat{f}$ invariant probability measures supported on $\Lambda^{f}$, and by $\mathcal{M}_{E}^{f}$ the subset of ergodic measures. According to a result of Rokhlin [8], for each $\mu \in \mathcal{M}$ there exists a unique $\hat{\mu} \in \mathcal{M}^{f}$ which is projected to $\mu$ by $\pi$. Moreover, if $\mu \in \mathcal{M}_{E}$, then $\hat{\mu} \in \mathcal{M}_{E}^{f}$.

We now discuss three important classes of measures for Axiom A endomorphisms. Qian and Zhu introduced the notion of an SRB measure for nonuniformly hyperbolic endomorphisms by formally extending the corresponding definition of diffeomorphisms to the inverse limit space (see [7]). Their definition adapted to the Axiom A case is as follows:

Definition 4. A measure $\mu \in \mathcal{M}$ is called SRB-measure if for every measurable partition $\eta$ of $M^{f}$ subordinate to the $W^{u}$-manifolds of $(f, \mu)$, we have that for $\hat{\mu}$-a.e. $\hat{x} \in \Lambda^{f}$,

$$
\pi\left(\hat{\mu}_{\hat{x}}^{\eta}\right)<<\lambda_{\hat{x}}^{u}
$$

where $\left\{\hat{\mu}_{\hat{x}}^{\eta}\right\}$ is a canonical system of conditional measures of $\hat{\mu}$ associated with $\eta, \pi\left(\hat{\mu}_{\hat{x}}^{\eta}\right)$ is the projection of $\hat{\mu}_{\hat{x}}^{\eta}$ under $\left.\pi\right|_{\eta(\hat{x})}$, and $\lambda_{\hat{x}}^{u}$ is the Lebesgue measure on $W^{u}(\hat{x})$ induced by the Riemannian structure of $M$.

We refer to [7] for more details.

Next, we introduce physical measures. We define the basin of a measure $\mu \in \mathcal{M}$ by

$$
\mathcal{B}(\mu)=\left\{x \in M: \frac{1}{n} \sum_{i=0}^{n-1} \delta_{f^{i}(x)} \rightarrow \mu \text { as } n \rightarrow \infty\right\} .
$$

Here we use convergence in the weak ${ }^{*}$ topology. We say that $\mu$ is a physical measure if there is a neighborhood $U$ of $\Lambda$ such that $\mathcal{B}(\mu) \cap U$ has full ${ }^{2}$ Lebesgue measure in $U$.

Finally, we recall that a measure $\mu \in \mathcal{M}$ satisfies Pesin's entropy formula if

$$
h_{\mu}(f)=\int_{\Lambda} \sum_{i} \lambda^{(i)}(x)^{+} m_{i}(x) d \mu
$$

\footnotetext{
${ }^{2}$ Sometimes in the literature it is required only that the Lebesgue measure of the intersection $\mathcal{B}(\mu) \cap U$ is positive. In our Theorem 8 either definition can be used.
} 
where $h_{\mu}(f)$ denotes the measure theoretic entropy of $f$ with respect to $\mu$ and $\lambda^{(i)}(x)^{+}$are the positive Lyapunov exponents at $x$ with multiplicity $m_{i}(x)$.

2.4. Stable sets and Bowen balls. We define the stable set of $\Lambda$ by

$$
W^{s}(\Lambda)=\left\{x \in M: d\left(f^{n}(x), \Lambda\right) \rightarrow 0 \text { for } n \rightarrow \infty\right\}
$$

and the local stable set of $\Lambda$ by

$$
W_{\varepsilon}^{s}(\Lambda)=\left\{x \in M: \operatorname{dist}\left(f^{k}(x), \Lambda\right)<\varepsilon \text { for all } k \in \mathbb{N}_{0}\right\} .
$$

It is an immediate consequence of the shadowing lemma that if $\varepsilon$ is sufficiently small, then there exists $\bar{\varepsilon}>0$ such that

$$
W_{\varepsilon}^{s}(\Lambda) \subset \bigcup_{x \in \Lambda} W_{\bar{\varepsilon}}^{s}(x)
$$

where $W_{\bar{\varepsilon}}^{s}(x)$ denotes the local stable manifold of size $\bar{\varepsilon}$ of $x \in \Lambda$. Furthermore, by choice of $\varepsilon$, the number $\bar{\varepsilon}$ can be chosen arbitrarily small.

For $x \in \Lambda, \varepsilon>0$ and $k \in \mathbb{N}$ we define the Bowen ball

$$
B(x, \varepsilon, k)=\left\{y \in M: d\left(f^{i}(x), f^{i}(y)\right)<\varepsilon, i=0, \ldots, k-1\right\},
$$

and

$$
B(\Lambda, \varepsilon, k)=\bigcup_{x \in \Lambda} B(x, \varepsilon, k)
$$

The following proposition immediately follows from Lemma 3.6 and Proposition 3.2 in [6].

Corollary 5. For all $\varepsilon, \delta>0$ small enough there exists $C_{\delta, \varepsilon} \geq 1$ such that for all $n \geq 1$, all $x \in \Lambda$ and all $y \in B(x, \max \{\varepsilon, \delta\}, n)$, we have that

$$
C_{\delta, \varepsilon}^{-1} \leq \frac{\operatorname{vol}(B(y, \delta, n))}{\operatorname{vol}(B(x, \varepsilon, n))} \leq C_{\delta, \varepsilon},
$$

where vol denotes the Lebesgue measure induced by the Riemannian structure on $M$.

2.5. Topological pressure. We will also need the topological pressure $P=$ $P_{\hat{f}}: C\left(\Lambda^{f}, \mathbb{R}\right) \rightarrow \mathbb{R}$, where $C\left(\Lambda^{f}, \mathbb{R}\right)$ denotes the space of continuous functions from $\Lambda^{f}$ to $\mathbb{R}$ (see [10] for the definition). A function $\varphi \in C\left(\Lambda^{f}, \mathbb{R}\right)$ is also called a potential. We are interested in the particular potential

$$
\phi^{u}: \Lambda^{f} \rightarrow \mathbb{R}
$$

defined by the formula

$$
\phi^{u}(\hat{x})=-\log \left|\operatorname{det} d_{x_{0}} f\right| E_{\hat{x}}^{u} \mid,
$$

where det $d_{x_{0}} f \mid E_{\hat{x}}^{u}$ denotes the Jacobian of the linear map $d_{x_{0}} f \mid E_{\hat{x}}^{u}$. The following result has been proven in [6] as Lemma 5.1. 
Proposition 6. Let $\varepsilon>0$ be small. Then

$$
\lim _{k \rightarrow \infty} \frac{1}{k} \log \left(\operatorname{vol}(B(\Lambda, \varepsilon, k))=P\left(\phi^{u}\right) \leq 0\right.
$$

We are now in position to prove the following main result of this section also modeled on the corresponding result of Bowen (see [2]) in the case of diffeomorphisms.

Theorem 7. The following are equivalent.

(i) $P\left(\phi^{u}\right)=0$;

(ii) $\operatorname{vol}\left(W_{\varepsilon}^{s}(\Lambda)\right)>0$ for arbitrary small $\varepsilon>0$;

(iii) $W_{\varepsilon}^{s}(\Lambda)$ has nonempty interior for arbitrary small $\varepsilon>0$;

(iv) $\Lambda$ is an attractor.

Proof. The implications (iv) $\Rightarrow($ iii $) \Rightarrow($ ii) are obvious. The implication (ii) $\Rightarrow(\mathrm{i})$ follows immediately from Proposition 6 and the observation that for all $k \in \mathbb{N}$, $B(\Lambda, \varepsilon, k) \supset W_{\varepsilon}^{s}(\Lambda)$. In order to prove that (i) $\Rightarrow($ iv) assume that $\Lambda$ is not an attractor. Fix $\gamma>0$ produced by Lemma 3. Since $f \mid \Lambda$ is uniformly hyperbolic, there exists $q \geq 0$ such that

$$
f^{q}\left(W_{\gamma / 4}^{u}(\hat{x})\right) \supset W_{\varepsilon}^{u}\left(\hat{f}^{q}(\hat{x})\right)
$$

for all $\hat{x} \in \Lambda^{f}$. Fix $n \geq 1$ and $\hat{E} \subset \Lambda^{f}$ such that $\pi(\hat{E})$ is a $(\gamma, n)$-separated set and $\left.\pi\right|_{\hat{E}}$ is one-to-one. Assuming that $n \geq 1$ is sufficiently large and using (6), we have that

$$
f^{n}\left(B\left(x_{0}, \gamma / 4, n\right)\right) \supset W_{\gamma / 4}^{u}\left(\hat{f}^{n}(\hat{x})\right) \text { and } f^{q}\left(W_{\gamma / 4}^{u}\left(\hat{f}^{n}(\hat{x})\right)\right) \supset W_{\varepsilon}^{u}\left(\hat{f}^{q+n}(\hat{x})\right) .
$$

Hence, applying Lemma 3, there exists $y(\hat{x}, n) \in B\left(x_{0}, \gamma / 4, n\right)$ such that

$$
f^{q+n}(y(\hat{x}, n)) \notin B(\Lambda, \gamma)
$$

By continuity of $f$ there is $\delta \in(0, \gamma / 4)$ so small that $d\left(f^{q}(z), f^{q}(y)\right)<\gamma / 2$ for all $y, z \in M$ with $d(z, y)<\delta$. Then

$$
B(y(\hat{x}, n), \delta, n) \subset B\left(x_{0}, \delta+\gamma / 4, n\right) \subset B\left(x_{0}, \gamma / 2, n\right)
$$

and

$$
f^{q+n}(B(y(\hat{x}, n), \delta, n)) \cap B(\Lambda, \gamma / 2)=\varnothing
$$


Hence $B(y(\hat{x}, n), \delta, n) \cap B(\Lambda, \gamma / 2, q+n)=\varnothing$. Using (19), (20) and Corollary 5, we therefore get

$$
\begin{aligned}
\operatorname{vol}(B(\Lambda, \gamma / 2, n)) & -\operatorname{vol}(B(\Lambda, \gamma / 2, n+q)) \geq \\
\geq & \operatorname{vol}\left(\bigcup_{\hat{x} \in \hat{E}} B(y(\hat{x}, n), \delta, n)\right)=\sum_{\hat{x} \in \hat{E}} \operatorname{vol}(B(y(\hat{x}, n), \delta, n)) \\
\geq & \sum_{\hat{x} \in \hat{E}} C_{\delta, \frac{3}{2} \gamma}^{-1} \operatorname{vol}\left(B\left(x_{0}, 3 \gamma / 2, n\right)\right) \\
\geq & C_{\delta, \frac{3}{2} \gamma}^{-1} \operatorname{vol}\left(\bigcup_{\hat{x} \in \hat{E}} B\left(\left(x_{0}, 3 \gamma / 2, n\right)\right)\right. \\
\geq & C_{\delta, \frac{3}{2} \gamma}^{-1} \operatorname{vol}(B(\Lambda, \gamma / 2, n))
\end{aligned}
$$

Consequently

$$
\operatorname{vol}(B(\Lambda, \gamma / 2, n+q)) \leq\left(1-C_{\delta, \frac{3}{2} \gamma}^{-1}\right) \operatorname{vol}(B(\Lambda, \gamma / 2, n))
$$

Applying Proposition 6 we therefore get $P\left(\phi^{u}\right) \leq \frac{1}{q} \log \left(1-C_{\delta, \frac{3}{2} \gamma}^{-1}\right)<0$ and we are done.

A part of this theorem was stated in [6] as Proposition 5.2, however the proof of the most difficult implication (i) $\Rightarrow$ (iv) is not included in [6].

\section{Characterization of SRB-measures}

In this section we provide equivalent conditions for the existence of an SRB measure on a basic set $\Lambda$ of a $C^{2}$ Axiom $\mathrm{A}$ endomorphism. In particular, we show that the existence of an SRB measure already follows from the fact that the stable set of $\Lambda$ has packing dimension $n$. This result has not been known before even in the case of hyperbolic diffeomorphisms. In the following, we denote by $\overline{\mathrm{BD}}(A)$ the upper box dimension of the set $A$. Recall that $\mathcal{C}_{f}$ denotes the set of critical points of $f$.

Theorem 8. Let $f$ be a $C^{2}$ Axiom A endomorphism on an n-dimensional compact Riemannian manifold $M$, and let $\Lambda$ be a basic set of $f$ such that $\mathcal{C}_{f} \cap \Lambda=\varnothing$. Then the following are equivalent.

(i) $\overline{\mathrm{BD}}\left(W_{\varepsilon}^{s}(\Lambda)\right)=n$ for arbitrary small $\varepsilon>0$;

(ii) $P\left(\phi^{u}\right)=0$;

(iii) $\Lambda$ is an attractor;

(iv) $f$ admits an $S R B$ measure on $\Lambda$;

(v) $f$ admits a physical measure on $\Lambda$;

(vi) there is an invariant measure on $\Lambda$ satisfying Pesin's entropy formula. 
Proof. First observe that (iii) $\Rightarrow($ iv $),(i i i) \Rightarrow(v)$ and $(i i i) \Rightarrow(v i)$ are known, see Corollary 1.1.2 of [7]. The equivalence $(i i) \Leftrightarrow(i i i)$ is proven in Theorem 7 . Moreover, $(i v) \Leftrightarrow(v i)$ is shown in [7], Theorem 1.1., and $(v i) \Leftrightarrow(i i)$ is Proposition 5.1 of [6]. The statement $(v) \Rightarrow(i)$ is trivial.

It remains to prove $(i) \Rightarrow(i i)$. We will prove that $P\left(\phi^{u}\right)<0$ implies $\overline{\mathrm{BD}}\left(W_{\varepsilon}^{s}(\Lambda)\right)<n$. Define $s=\log \left(\max \left\{\left\|d_{x} f\right\|: x \in \Lambda\right\}\right)$. Without loss of generality, we may assume that $E^{u}$ is non-trivial, because otherwise the result is trivial. Hence $s>0$.

Claim. If $P\left(\phi^{u}\right)<0$ and $\varepsilon>0$ is small enough, then

$$
\overline{\mathrm{BD}}\left(W_{\varepsilon}^{s}(\Lambda)\right)<n+\frac{P\left(\phi^{u}\right)}{s}<n .
$$

Note that the right-hand side inequality is trivial. Let $\delta>0$. By continuity there exist $\varepsilon>0$ and such that for all $x \in B\left(W_{\varepsilon}^{s}(\Lambda), \varepsilon\right)=\{y \in M: \exists z \in$ $\left.W_{\varepsilon}^{s}(\Lambda), d(y, z)<\varepsilon\right\}$ we have

$$
\left\|d_{x} f\right\|<\exp (s+\delta)
$$

It follows from Proposition 6 that for $\varepsilon$ sufficiently small,

$$
P\left(\phi^{u}\right)=\lim _{k \rightarrow \infty} \frac{1}{k} \log (\operatorname{vol}(B(\Lambda, 2 \varepsilon, k))),
$$

where $B(\Lambda, 2 \varepsilon,, k)=\bigcup_{x \in \Lambda} B(x, 2 \varepsilon, k)$ (see (15)). Thus, if $k \in \mathbb{N}$ is large enough, then

$$
\operatorname{vol}(B(\Lambda, 2 \varepsilon, k))<\exp \left(k\left(P\left(\phi^{u}\right)+\delta\right)\right) .
$$

For all $k \in \mathbb{N}$ we define real numbers

$$
r_{k}=\frac{\varepsilon}{\exp (s+\delta)^{k}}
$$

and neighborhoods $B_{k}=B\left(W_{\varepsilon}^{s}(\Lambda), r_{k}\right)$ of $W_{\varepsilon}^{s}(\Lambda)$. Let $y \in B_{k}$. Then there exists $x \in W_{\varepsilon}^{s}(\Lambda)$ with $d(x, y)<r_{k}$. An elementary induction argument in combination with the mean-value theorem implies $d\left(f^{i}(x), f^{i}(y)\right)<\varepsilon$ for all $i \in\{0, \ldots, k-1\}$. Using (14) and making $\varepsilon$ smaller if necessary, we can assure that $x$ is contained in the local stable manifold of size $\varepsilon$ of a point in $\Lambda$. It follows that $y \in B(\Lambda, 2 \varepsilon, k)$. Hence $B_{k} \subset B(\Lambda, 2 \varepsilon, k)$. Therefore, (24) implies that

$$
\operatorname{vol}\left(B_{k}\right)<\exp \left(k\left(P\left(\phi^{u}\right)+\delta\right)\right)
$$

for sufficiently large $k$. Let us recall that for $t \in[0, n]$ the $t$-dimensional upper Minkowski content of a relatively compact set $A \subset M$ is defined by

$$
M^{* t}(A)=\limsup _{\rho \rightarrow 0} \frac{\operatorname{vol}\left(A_{\rho}\right)}{(2 \rho)^{n-t}}
$$


where $A_{\rho}=\{x \in M: \exists y \in A: d(x, y) \leq \rho\}$. Let $t \in[0, n]$ and $\rho_{k}=\frac{r_{k}}{2}$ for all $k \in \mathbb{N}$. Then we have

$$
\begin{aligned}
M^{* t}\left(W_{\varepsilon}^{s}(\Lambda)\right) & =\limsup _{\rho \rightarrow 0} \frac{\operatorname{vol}\left(W_{\varepsilon}^{s}(\Lambda)_{\rho}\right)}{(2 \rho)^{n-t}} \\
& \leq \limsup _{k \rightarrow \infty} \frac{\operatorname{vol}\left(W_{\varepsilon}^{s}(\Lambda)_{\rho_{k}}\right)}{\left(2 \rho_{k+1}\right)^{n-t}} \\
& \leq \limsup _{k \rightarrow \infty} \frac{\operatorname{vol}\left(B_{k}\right)}{\left(r_{k+1}\right)^{n-t}} \\
& \leq \frac{\exp (s+\delta)^{n-t}}{\varepsilon^{n-t}} \lim _{k \rightarrow \infty}\left(k \exp (s+\delta)^{n-t} \exp \left(P\left(\phi^{u}\right)+\delta\right)\right) .
\end{aligned}
$$

Let $t>n+\frac{P\left(\phi^{u}\right)+\delta}{s+\delta}$. Then $\exp (s+\delta)^{n-t} \exp \left(P\left(\phi^{u}\right)+\delta\right)<1$. This implies $M^{* t}\left(W_{\varepsilon}^{s}(\Lambda)\right)=0$, in particular, $t \geq \overline{\mathrm{BD}}\left(W_{\varepsilon}^{s}(\Lambda)\right)$. Since $\delta$ can be chosen arbitrarily small, the claim follows. This completes the proof of the theorem.

Remark. A similar technique as in the proof of $(i) \Rightarrow(i i)$ was used in [9] in order to estimate the box dimension of hyperbolic sets of $C^{2}$ diffeomorphisms.

Combining Theorem 8 with Corollary 1.1.2 of [7] we obtain the following uniqueness result.

Corollary 9. Let $f$ be a $C^{2}$ Axiom A endomorphism on an n-dimensional compact Riemannian manifold $M$, and let $\Lambda$ be a basic set of $f$ such that $\mathcal{C}_{f} \cap \Lambda=\varnothing$. Let $\mu \in \mathcal{M}$. Then the following are equivalent:

(i) $\mu$ is an $S R B$ measure;

(ii) $\mu$ is a physical measure;

(iii) $\mu$ satisfies Pesin's entropy formula.

Moreover, in each of these cases, the measure $\mu$ is unique, and $\mu \in \mathcal{M}_{E}$.

The postcritical set of $f$ is defined by $\operatorname{PC}(f)=\bigcup_{n \in \mathbb{N}_{0}} f^{n}\left(\mathcal{C}_{f}\right)$. Assuming that the closure of the postcritical set of $f$ is disjoint from $\lambda$, we obtain an equivalent condition for the existence of an SRB measure in terms of the packing dimension of the stable set of $\Lambda$.

Theorem 10. Let $f$ be a $C^{2}$ Axiom $A$ endomorphism on an n-dimensional compact Riemannian manifold $M$, and let $\Lambda$ be a basic set of $f$ such that $\overline{\mathrm{PC}(f)} \cap$ $\Lambda=\varnothing$. Then each of the conditions in Theorem 8 is equivalent to either of the following conditions.

(i) $\operatorname{PD}\left(\mathrm{W}^{\mathrm{s}}(\Lambda)\right)=\mathrm{n}$

(ii) $W^{s}(\Lambda)$ has positive Lebesgue measure.

Proof. By hypothesis, $\mathcal{C}_{f} \cap \Lambda=\varnothing$. Therefore, Theorem 8 applies. Assume $f$ admits a physical measure on $\Lambda$ (condition $(v)$ of Theorem 8$)$. Thus, $W^{s}(\Lambda)$ has positive Lebesgue measure, which implies that $\operatorname{PD}\left(\mathrm{W}^{\mathrm{s}}(\Lambda)\right)=\mathrm{n}$. Observe that

$$
\bigcup_{n \in \mathbb{N}} f^{-n}\left(W_{\varepsilon}^{s}(\Lambda)\right)=W^{s}(\Lambda)
$$


Using that $\overline{\mathrm{PC}(f)} \cap \Lambda=\varnothing$, we are able to show that $\mathrm{PD}\left(\mathrm{f}^{-\mathrm{n}}\left(\mathrm{W}_{\varepsilon}^{\mathrm{s}}(\Lambda)\right)\right)=$ $\operatorname{PD}\left(\mathrm{W}_{\varepsilon}^{\mathrm{s}}(\Lambda)\right)$ for all $n \in \mathbb{N}$. Since the the packing dimension is stable under countable unions, it follows that $\operatorname{PD}\left(\mathrm{W}_{\varepsilon}^{\mathrm{s}}(\Lambda)\right)=\mathrm{n}$. Therefore, condition $(i)$ of Theorem 8 follows from the fact that the packing dimension of a set is bounded above by the upper box dimension of the set.

Remark. Theorem 10 remains true if we replace $\overline{\mathrm{PC}(f)} \cap \Lambda=\varnothing$ with the condition that $\mathcal{C}_{f}$ is a discrete set not intersecting $\Lambda$.

As a consequence of the proofs of Theorems 8 and 10 we obtain the following.

Corollary 11. Let $f$ be a $C^{2}$ Axiom $A$ endomorphism on an $n$-dimensional compact Riemannian manifold $M$, and let $\Lambda$ be a basic set of $f$ such that $\overline{\mathrm{PC}(f)} \cap$ $\Lambda=\varnothing$. Define

$$
s=\log \left(\max \left\{\left\|d_{x} f\right\|: x \in \Lambda\right\}\right)
$$

Then

$$
\mathrm{PD}\left(\mathrm{W}^{\mathrm{s}}(\Lambda)\right) \leq \mathrm{n}+\frac{\mathrm{P}\left(\phi^{\mathrm{u}}\right)}{\mathrm{s}}
$$

In particular, if $\Lambda$ is not an attractor, then $\mathrm{PD}\left(\mathrm{W}^{\mathrm{s}}(\Lambda)\right)<\mathrm{n}$.

\section{References}

[1] R. Bowen, A horseshoe with positive measure, Invent. Math. 29 (1975), 203-204.

[2] R. Bowen, Equilibrium states and the ergodic theory of Anosov diffeomorphisms, Lecture Notes in Mathematics 470, Springer, 1975.

[3] R. Bowen, D. Ruelle, The ergodic theory of Axiom A flows, Invent. Math. 29 (1975), 181-202.

[4] M. Jonsson, Hyperbolic dynamics of endomorphisms, unpublished manuscript, avail. at: http://www.math.lsa.umich.edu/ mattiasj

[5] F. Przytycki, Anosov endomorphisms, Studia Math. 58 (1976), 249-285.

[6] M. Qian, Z.S. Zhang, Ergodic theory for Axiom A endomorphisms, Ergodic Theory Dynam. Systems 15 (1995), 161-174.

[7] M. Qian, S. Zhu, SRB measures and Pesin's entropy formula for endomorphisms, Trans. Amer. Math. Soc. 354 (2002), 1453-1471.

[8] V.A. Rokhlin, Lectures on the on the theory of entropy of transformations with invariant measures, Russ. Math. Surveys, 22 (1967), 1-54.

[9] R. Shafikov, C. Wolf, Stable sets, hyperbolicity and dimension, Discrete and Cont. Dyn. Sys, to appear.

[10] P. Walters, An introduction to ergodic theory, Graduate Texts in Mathematics 79, Springer, 1981.

[11] L.-S. Young, What are SRB measures, and which dynamical systems have them? Dedicated to David Ruelle and Yasha Sinai on the occasion of their 65th birthdays, J. Statist. Phys. 108 (2002), 733-754. 
Department of Mathematics, University of North Texas, P.O. Box 311430, DenTON, TX 76203-1430, USA

E-mail address: urbanski@unt.edu

$U R L:$ http://www.math.unt.edu/ũrbanski

Department of Mathematics, Wichita State University, Wichita, KS 67260, USA

E-mail address: cwolf@math.wichita.edu 